\title{
Transitivity and Minimality of Sets
}

\author{
Dana Mawlood Mohammed* \\ Institute of Training and Educational Development, Sulaimani \\ *Corresponding author: Danamath82@gmail.com
}

Received August 11, 2018; Revised September 15, 2018; Accepted October 22, 2018

\begin{abstract}
In this paper, we have study some concepts of minimal open, closed sets and minimal functions. Further, we have shown that these properties preserved under conjugate maps.
\end{abstract}

Keywords: minimal open sets, minimal functions, transitivity

Cite This Article: Dana Mawlood Mohammed, "Transitivity and Minimality of Sets." Turkish Journal of Analysis and Number Theory, vol. 6, no. 5 (2018): 149-151. doi: 10.12691/tjant-6-5-5.

\section{Introduction}

Let $(X, \tau)$ be a compact topological space. All maps under consideration are supposed to be continuous. The set of all continuous maps $\mathrm{f}: \mathrm{X} \rightarrow \mathrm{X}$ is denoted by $\mathrm{C}(\mathrm{X})$. By a system $(X, f)$, we mean a compact topological space (phase space) $X$ and $f \in C(X)$. In a topological space a trajectory consists of a sequence of points $\left(x, f(x), f^{2}(x), \ldots\right)$, and can possibly contain additional attributes $a$ measured at each point. Trajectories can be generated by moving objects but also by moving phenomena, e.g. measurement points on a hill slide. The pointscan be captured at regular intervals or irregularly A point $x \in X$ "moves," its trajectory [1] being the sequence $x, f(x), f^{2}(x), \ldots$, where $f^{n}$ is the $n$th iteration of $f$.

The point $f^{n}(x)$ is the position of the point $x$ after $n$ units of time. The set of points of the trajectory of $x$ under $f$ is called the orbit of $x$, denoted by $O_{f}(x)$. A map $\mathrm{f} \in \mathrm{C}(\mathrm{X})$ is (topologically) transitive if for any two nonempty open sets $\mathrm{U}$ and $\mathrm{V}$ in $\mathrm{X}$, there is a nonnegative integer $\mathrm{n}$ such that $f^{n}(U) \cap V \neq \varphi$. If $\mathrm{X}$ has no isolated points then this definition is equivalent to the existence of a dense orbit, i.e. $\mathrm{Cl}\left(O_{f}(x)\right)=X$. If every orbit of $\mathrm{f}$ is dense, the map $f$ is called minimal. Denote by $T(X)$ the set of transitive self-maps of the space $\mathrm{X}$. A minimal map $f$ is necessarily surjective if $X$ is assumed to be Hausdorff and compact.

Now, to study the existence of minimal sets, given a system $(X, f)$, a set $A \subseteq X$ is called a minimal set if it is non-empty, closed and invariant and if no proper subset of $A$ has these three properties. So, $A \subseteq X$ is a minimal set if and only if $(A, f \mid A)$ is a minimal system. A system $(X, f)$ is minimal if and only if $\mathrm{X}$ is a minimal set in
$(X, f)$. The basic fact discovered by G. D. Birkhoff is that in any compact system $(X, f)$ there are minimal sets. This follows immediately from the Zorn's lemma. Since any orbit closure is invariant, we get that any compact orbit closure contains a minimal set. This is how compact minimal sets may appear in non-compact spaces. Two minimal sets in $(X, f)$ either are disjoint or coincide. A minimal set $\mathrm{A}$ is strongly $f$-inveriant, i.e. $f(A)=A$. Provided it is compact Hausdorff

\section{Preliminaries and Definitions}

\section{Definition 2.1}

1. (Minimal Hausdorff spaces) [3]

A topological space $(X, \tau)$ is said to be minimal Hausdorff if $\tau$ is Hausdorff and there exists no Hausdorff topology on $\mathrm{X}$ strictly weaker than $\tau$. Thus this minimality property is topological.

2. Two topological spaces $(X, \tau)$ and $\left(Y, \tau_{1}\right)$ are called homeomorphic [5] if there exists a one-to-one onto function $f:(X, \tau) \rightarrow\left(Y, \tau_{1}\right)$ such that $f$ and $f^{-1}$ are both continuous.

3. Two topological systems $f: X \rightarrow X$ and $g: Y \rightarrow Y$ are said to be topologically conjugate if there is a homeomorphism $h: X \rightarrow Y$ such that $h \circ f=g \circ h$. We will call $\mathrm{h}$ topological Conjugacy. Thus, the two topological systems with their respective function acting on them share the same dynamics(see the following diagram )

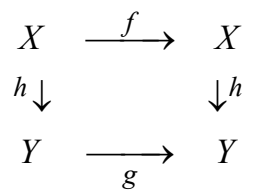

Definition 2.2 (minimal open set). Recall that a proper non empty open subset $U$ of a topological space $X$ is said 
to be a minimal open set, if any open set which is contained in $\mathrm{U}$ is empty set or $\mathrm{U}$.

Definition 2.3 Recall that a proper non empty closed subset $\mathrm{F}$ of a topological space $\mathrm{X}$ is said to be a minimal closed set if any closed set which is contained in $F$ is empty set or F.

\section{Definition 2.4}

A system $(X, f)$ is called minimal if $X$ does not contain any non-empty, proper, closed $f$-inveriant subset. In such a case we also say that the map $f$ itself is minimal. Thus, one cannot simplify the study of the dynamics of a minimal system by finding its non- trivial closed subsystems and studying first the dynamics restricted to them.

\section{Proposition 2.5}

Let $f: X \rightarrow X$ be continuous function. The following are equivalent:

1. $f$ is minimal.

2. The only closed invariant sets of $X$ are $X$ itself and the empty set.

3. For any non-empty open subset $U \subset X$, then $X=\bigcup_{k=0}^{\infty} f^{-k}(U)$.

\section{Proof:}

$(1) \Rightarrow(2)$

Suppose that $C \subset X$ is a non-empty closed invariant set. Let $x \in C$. Then since $\mathrm{C}$ is invariant, $O_{f}(x) \subset C$. Since $\mathrm{C}$ is closed, so $\mathrm{Cl}\left(O_{f}(x)\right) \subset C$, but $X=C l\left(O_{f}(x)\right)$ since the orbit $O_{f}(x)$ is dense, this means $X \subset C$. Thus $\mathrm{X}=\mathrm{C}$.

(2) $\Rightarrow$ (3), let $U \subset X$ be a non-empty open set. Put $C=X \backslash \bigcup_{k=0}^{\infty} f^{-k}(U)$ then $\mathrm{C}$ is closed and invariant. Since $C \neq X$, by (2) we must have $C=\varphi$.

(3) $\Rightarrow$ (1), let $x \in X$ and let $U$ be an arbitrary non-empty open subset. Then by (3), $x \in f^{-k}(U)$ for some $k \geq 0$. Thus $f^{k}(x) \in U$, and hence $O_{f}(x) \cap U \neq \varphi$. Since $\mathrm{U}$ was arbitrary, $O_{f}(x)$ is dense, i.e. $\mathrm{f}$ is minimal.

Definition 2.6 (minimal) Let $\mathrm{X}$ be a topological space And $f$ be continuous map on $\mathrm{X}$. Then $(X, f)$ is called minimal system (or $\mathrm{f}$ is called minimal map on $\mathrm{X}$ ) if one of the three equivalent conditions hold:

(1) The orbit of each point in $\mathrm{X}$ is dense in $\mathrm{X}$

(2) $\mathrm{Cl}\left(O_{f}(x)\right)=X$ for each $\mathrm{x} \in \mathrm{X}$.

(3) Given $x \in X$ and a nonempty open $U$ in $X$, there exists $n \in \mathbf{N}$ such that $f^{n}(x) \in U$.

Definition 2.7 A subset $M$ of $X$ is said to be minimal under provided that $\mathrm{M}$ is non-empty, closed and invariant, that is $f(M) \subset M$, and no proper subset of $\mathrm{M}$ has all these properties.

Theorem 2.8 [4] Any two minimal sets must have empty intersection.
Proof: Let $M_{1}$ and $M_{2}$ be two distinct minimal sets, and suppose that $A=M_{1} \cap M_{2} \neq \varphi$. Then $\mathrm{A}$ is closed, and fore very a $\in \mathrm{A}$ and every $\mathrm{n} \in \mathrm{N}, f^{n}(a) \in M_{1} \cap M_{2}$, so A is invariant. But then $\mathrm{A}$ is a proper subset of both $M_{1}$ and $M_{2}$ which is closed, invariant and non-empty, contradicting the fact that $M_{1}$ and $M_{2}$ are minimal.

Definition 2.9 Two topological systems $f: X \rightarrow X$ and $g: Y \rightarrow Y$ are said to be topologically conjugate if there is a homeomorphism $h: X \rightarrow Y$ such that $h \circ f=g \circ h$. We will call $\mathrm{h}$ a topological Conjugacy.

We have stated a new proposition as follows:

Proposition $\mathbf{2 . 1 0}$ if $f: X \rightarrow X$ and $g: Y \rightarrow Y$ are topologically conjugated by $h: X \rightarrow Y$. Then $A$ is a minimal open set in $\mathrm{X}$ if and only if $h(A)$ is a minimal open set in $\mathrm{Y}$.

Definition 2.11 Let $X$ be a topological space, and $f: X \rightarrow X$ a continuous map. We say $\mathrm{f}$ is (topologically) transitive if for any nonempty open sets $U . V \subset X$ there exists $\mathrm{n}>0$ such that $V \cap f^{n}(U) \neq \varphi$. We say $\mathrm{f}$ is strongly transitive [3] if for any nonempty open set $U \subset X, \quad X=\bigcup_{k=0}^{s} f^{k}(U)$ for some $\mathrm{s}>0$. For more knowledge see [4].

Definition 2.12 A subset $S$ of $X$ is called $\wedge$-set if it is the intersection of open sets containing $\mathrm{S}$.

Definition 2.13 Recall that a subset of a topological space $(X, \tau)$ is called $\lambda$-closed set if $A=S \cap C$ where $\mathrm{S}$ is $\wedge$ set and $C$ is a closed set.

Proposition 2.14 Let $(X, \tau)$ be topological space and $\mathrm{A}$ be a nonempty $\lambda$-closed $f$-invariant set of $\mathrm{X}$. Then $\mathrm{A}$ is a $\lambda$-type transitive set of $(X, f)$ if and only if $(A, f)$ is $\lambda$ type transitive.

Proof:

$\Rightarrow$ Let $V_{1}$ and $U_{1}$ be two nonempty $\lambda$-open subsets of A. For a nonempty $\lambda$-open subset $U_{1}$ of $\mathrm{A}$, there exists a $\lambda$ - open set $\mathrm{U}$ of $\mathrm{X}$ such that $U_{1}=U \cap A$ Since $\mathrm{A}$ is a $\lambda$-type transitive set of $(X, f)$, there exists $\mathrm{n} \in \mathrm{N}$ such that $f\left(V_{1}\right) \cap U \neq \varphi$. Moreover, $\mathrm{A}$ is invariant, i.e., $f(A) \subset A$, which implies that $f(A) \subset A$. Therefore, $f\left(V_{1}\right) \cap A \cap U \neq \varphi$, i.e. $f\left(V_{1}\right) \cap U_{1} \neq \varphi$. This shows that $(A, f)$ is $\lambda$ - type transitive.

$\Leftarrow)$ Let $V_{1}$ be a nonempty $\lambda$-open set of $\mathrm{A}$ and $\mathrm{U}$ be a nonempty $\lambda$-open set of $\mathrm{X}$ with $A \cap U \neq \varphi$, Since $\mathrm{U}$ is an $\lambda$-open set of $\mathrm{X}$ and $A \cap U \neq \varphi$, it follows that $\mathrm{U} \cap \mathrm{A}$ is a nonempty $\lambda$-open set of A. Since $(A, f)$ is topologically $\lambda$-type transitive, there exists $\mathrm{n} \in \mathrm{N}$ such that $f\left(V_{1}\right) \cap(A \cap U) \neq \varphi$, which implies that $f\left(V_{1}\right) \cap U \neq \varphi$. This shows that $A$ is a $\lambda$-type transitive set of $(X, f)$.

Theorem 2.15 Let $X$ be a non-empty $\lambda$-compact Hausdorff space. Then the intersection of a countable collection of $\lambda$-open $\lambda$-dense subsets of $X$ is $\lambda$-dense in $X$. 
Definition 2.16 Let $(X, \tau)$ be a topological space. Recall that subset $\mathrm{A}$ of $\mathrm{X}$ is called $\lambda$-dense in $\mathrm{X}$ if $\lambda \mathrm{Cl}(\mathrm{A})=\mathrm{X}$.

Corollary 2.17 A subset $\mathrm{A}$ of a space $(X, \tau)$ is $\lambda$-dense if and only if $A \cap U \neq \varphi$ for all $U \in \tau^{\lambda}$ other than $U=\varphi$.

Proof: If $\mathrm{A}$ is $\lambda$-dense set in $\mathrm{X}$, then by definition, $\lambda C l(A)=X$, and let $\mathrm{U}$ be a non-empty $\lambda$-open set in $\mathrm{X}$. Suppose that $\mathrm{A} \cap \mathrm{U}=\phi$. Therefore $B=U^{c}$ is $\lambda$-closed and $A \subset U^{C}=B$. So, $\lambda C l(A) \subset \lambda C l(B)$, i.e. $\lambda C l(A) \subset B$, but $\lambda C l(A)=X$, so $\mathrm{X} \subset \mathrm{B}$, this contradicts $\mathrm{U} \neq \varphi$.

Theorem 2.18 Let $(X, \tau)$ be a non-empty $\lambda$-compact Hausdorff space and $f: X \rightarrow X$ is $\lambda$-irresolute map and that $\mathrm{X}$ is $\lambda$-type separable. Suppose that $\mathrm{f}$ is topologically $\lambda$-type transitive. Then there is an element $x \in X$ such that the orbit $O_{f}(x)=\left\{x, f(x), f^{2}(x), \ldots f^{n}(x), \ldots ..\right\}$ is $\lambda$-dense in $\mathrm{X}$.

Proof: Let $\mathrm{B}=\left\{U_{i}\right\} i=1,2,3, \ldots$ be a countable basis for the $\lambda$-topology of $X$. For each i, let $O_{i}=\left\{x \in X: f^{n}(x) \in U_{i}\right.$ for some $\left.n \geq 0\right\}$

Then, clearly $O_{i}$ is $\lambda$-open and $\lambda$-dense. It is $\lambda$-open since $\mathrm{f}$ is $\lambda$-irresolute, so, $O_{i}=\bigcup_{i=1}^{\infty} f^{-1}\left(U_{i}\right)$ is $\lambda$ - open and $\lambda$-dense since $\mathrm{f}$ is topological $\lambda$ - transitive map. Further, for every $\lambda$-open set $\mathrm{V}$, there is $\mathrm{n}>0$, such that $f^{n}(V) \cap U_{i} \neq \varphi$, since $\mathrm{f}$ is $\lambda$ - transitive.

Now, apply theorem 2.15 to the countable $\lambda$-dense set $\left\{O_{i}\right\}$ to say that $\bigcap_{i=0}^{\infty} O_{i}$ is $\lambda$-dense and so non-empty. Let $y \in \bigcap_{i=0}^{\infty} O_{i}$. This means that, for each $\mathrm{i}$, there is a positive integer $\mathrm{n}$ such that $f^{n}(y) \in U_{i}$ for each i. By corollary 2.17, this implies that $O_{f}(x)$ is $\lambda$-dense in $X$.

\section{References}

[1] Bruckner A. M., Hu Thakyin On scrambled sets for chaotic functions,Trans. Amer. Math. Soc. 301.1 (1987), 289-297.

[2] Anuradha $\mathrm{N}^{1}$ and Baby Chacko ${ }^{2}$, On Minimal Regular Open Sets and Maps in Topological Spaces, Journal of Computer and Mathematical Sciences, Vol.6(4), April 2015. Pp. 182-192.

[3] A. KAMEYAMA, TOPOLOGICAL TRANSITIVITY AND STRONG TRANSITIVITY, Acta Math. Univ. Comenianae Vol. LXXI, 2(2002), pp. 139-145.

[4] Mohammed Nokhas Murad Kaki, Introduction to Topological Dynamical Systems II, Lambert academic publisher, Germany.

[5] P.S. Aleksandrov, H. HopfTopologieSpringer Verlag, Berlin (1935). 